\title{
Risco de suicídio e comorbidades psiquiátricas no transtorno de ansiedade generalizada
}

\author{
Risk of suicide and psychiatric comorbidities in generalized anxiety disorder
}

Juarez Roberto de Oliveira Vasconcelos', Alice Peixoto da Silva Lôbo', Valfrido Leão de Melo Neto

\section{RESUMO}

Objetivo: Investigar o risco de suicídio nos pacientes com transtorno de ansiedade generalizada (TAG). Métodos: Estudo transversal com 253 pacientes dos ambulatórios de Psiquiatria, Nefrologia e Cardiologia do Hospital Universitário da Universidade Federal de Alagoas. O instrumento utilizado foi o MINI 5.0.0 (Mini-International Neuropsychiatric Interview). Resultados: Os indivíduos com TAG corresponderam a 16,6\% da amostra, sendo $30,8 \%$ nos pacientes entrevistados no ambulatório de psiquiatria e 11,3\% nos pacientes entrevistados nos demais ambulatórios. A maioria é do sexo feminino (83,3\%), casada $(53,8 \%)$, sendo a média de idade de $44( \pm 12,2)$ anos. Houve comorbidade psiquiátrica em $88,1 \%$ dos entrevistados; $54,8 \%$ possuíam duas ou mais e 33,3\% tinham apenas uma. A mais prevalente foi depressão maior (53,7\%), seguida de distimia $(21,4 \%)$ e depressão recorrente $(14,6 \%)$. O risco de suicídio foi observado em $54,8 \%$ dos indivíduos com TAG. Conclusão: A prevalência de TAG foi significativamente maior que na população geral, principalmente no ambulatório de psiquiatria. A maioria dos pacientes apresentou comorbidade psiquiátrica, especialmente transtornos de humor, além de risco de suicídio. O TAG mostrou elevada correlação com transtornos de humor e significativa associação com o risco de suicídio.

\begin{abstract}
Objective: Investigate the risk of suicide in patients with generalized anxiety disorder (GAD) Methods: Cross-sectional study with 253 outpatients from the Psychiatry, Nephrology, and Cardiology units of the University Hospital of the Federal University of Alagoas. The instrument used was MINI 5.0.0 (Mini-International Neuropsychiatric Interview). Results: GAD was present in $16.6 \%$ of the sample, which were $30.8 \%$ from the psychiatric unit and $11.3 \%$ in the outpatients of the other clinics. The majority were females (83.3\%), married (53.8\%), with a mean age of $44( \pm 12.2)$ years old. There was psychiatric comorbidity in $88.1 \%$ of the evaluated patients, of which $54.8 \%$ had two or more and $33.3 \%$ had only one. Major depression was the most prevalent (53.7\%), followed by dysthymia (21.4\%) and recurrent depression (14.6\%). The risk of suicide was observed in $54.8 \%$ of subjects with GAD. Conclusion: The prevalence of GAD in outpatient clinical samples was significantly higher than in the general population, mainly in the psychiatric clinic. The majority of the patients presented psychiatric comorbidity, especially mood disorders besides presenting risk of suicide. GAD showed high correlation with mood disorders and significant association with risk of suicide.
\end{abstract}

1 Universidade Federal de Alagoas (UFAL), Faculdade de Medicina (Famed).

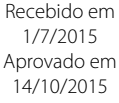

DOI: 10.1590/0047-2085000000087
Endereço para correspondência: Valfrido Leão de Melo Neto Universidade Federal de Alagoas, Maceió, AL, Brasil E-mail: valfrido@gmail.com 


\section{INTRODUÇÃO}

O transtorno de ansiedade generalizada (TAG) é um transtorno psiquiátrico que se caracteriza pela preocupação excessi$v^{2}{ }^{1}$. De acordo com o DSM-5, para ser diagnosticada, deve durar pelo menos seis meses e ser acompanhada de pelo menos três dos seguintes sintomas: inquietação, irritabilidade, fatigabilidade, perturbação do sono, tensão muscular e/ ou dificuldade de concentração².

O National Comorbidity Survey Replication (NCS-R), principal estudo epidemiológico norte-americano, que avaliou 9.282 pessoas da comunidade, encontrou prevalência de 5,7\% de TAG durante a vida ${ }^{3}$, enquanto estudos europeus chegaram a relatar prevalência de até 6,9\% ${ }^{4}$. O Estudo Epidemiológico dos Transtornos Mentais São Paulo Megacity (2009), que avaliou 5.037 indivíduos da região metropolitana de São Paulo, revelou que 19,9\% apresentam algum transtorno de ansiedade e 2,3\% possuem TAG ${ }^{5}$; essas proporções, segundo esse mesmo estudo, seriam semelhantes às encontradas em outros grandes centros urbanos brasileiros. O NCS-R revela também uma frequência duas vezes superior em mulheres que em homens, sendo a maior parte de viúvas e divorciadas'. A maioria dos casos inicia-se entre 20 e 47 anos, com caráter crônico e recorrente'1. Em relação à escolaridade, os estudos são bastante discordantes, alguns referindo predomínio naqueles com níveis mais altos de instrução, enquanto outros nos níveis mais baixos ${ }^{6}$. A etiologia é multifatorial, consistindo em fatores de risco tanto estressores psicossociais (problemas no trabalho, humilhações, perdas e situações de perigo) como fatores genéticos ${ }^{7}$. A taxa de herdabilidade varia entre $14,3 \%$ e $49 \%$, a depender dos critérios diagnósticos utilizados ${ }^{8}$.

OTAG é um transtorno com altas taxas de comorbidade, destacando-se transtornos de humor e outros transtornos de ansiedade 9 . O paciente com TAG apresenta risco 33 vezes superior ao da população geral de apresentar transtorno depressivo maior e 20 vezes maior de apresentar transtorno de pânico ${ }^{4}$. A presença de tais comorbidades complica o curso, tornando a recuperação mais difícil ${ }^{4}$. Estudo recente indica que, em $68 \%$ dos casos, o TAG precede os transtornos depressivos ou surge concomitantemente a eles, funcionando inclusive como fator de risco ${ }^{10}$.

O TAG também guarda relação com transtornos não psiquiátricos como doenças cardiovasculares e renais ${ }^{7}$. É observado em $20 \%$ dos cardiopatas do sexo masculino, precedendo doenças cardíacas em 62\% dos casos? ${ }^{7}$. Ocorre em 17\% dos pacientes portadores de doença renal crônica ${ }^{11}$. A associação com o TAG também torna o prognóstico mais reservado em ambas as situações ${ }^{12}$.

Sabe-se ainda da forte associação do comportamento suicida com transtornos psiquiátricos (88\%-98\%), inclusive os de ansiedade ${ }^{13}$. Faucett et al., em estudo com 954 pacientes acompanhados por 10 anos, foram dos primeiros a estabelecer a relação entre suicídio e ansiedade. Ocorreram 32 suicídios durante o período avaliado, sendo registrada forte correlação com sintomas ansiosos ${ }^{14}$. Entretanto, poucos estudos associam especificamente o TAG (e suas comorbidades) ao comportamento suicida. Entre os pacientes com transtorno bipolar e TAG comórbidos, $62 \%$ já relataram ter tentado suicídio, enquanto apenas 22\% daqueles sem TAG o fizeram ${ }^{2}$. Pacientes deprimidos com TAG comórbido apresentam três vezes mais chances de tentar suicídio que aqueles com depressão sem tal comorbidade ${ }^{15}$. Dessa maneira, torna-se notória a necessidade de mais pesquisas acerca desse tema.

Sendo assim, o presente estudo propõe a investigação de comorbidades psiquiátricas, risco de suicídio e suas correlações em pacientes com TAG atendidos em diferentes serviços de um hospital geral.

\section{MÉTODOS}

\section{Tipo de estudo}

Estudo de corte transversal.

\section{Local e amostra}

Foram selecionados consecutivamente, por conveniência, 253 pacientes, pertencentes aos ambulatórios de Psiquiatria, Nefrologia e Cardiologia do Hospital Universitário Professor Alberto Antunes (HUPAA) da Universidade Federal de Alagoas (UFAL).

\section{Instrumentos}

- Questionário sociodemográfico breve, elaborado especificamente para esta pesquisa, que investigava: idade, sexo, escolaridade e estado civil.

- MINI 5.0.0 (Mini-International Neuropsychiatric Interview): uma entrevista diagnóstica padronizada breve (15-30 minutos), compatível com os critérios do DSM-IV, destinada à atenção primária e pesquisa, foi utilizada com o objetivo de investigar os principais transtornos psiquiátricos de eixo I, além de transtorno de personalidade antissocial e risco de suicídio $^{16}$.

\section{Procedimentos}

A pesquisa foi aprovada pelo comitê de ética da UFAL, seguindo os preceitos da Declaração de Helsinki, sendo garantida a confidencialidade das informações. O MINI 5.0.0 foi utilizado por estudantes de Medicina supervisionados por psiquiatra experiente, após treinamento inicial. O objetivo do uso desse instrumento foi investigar a prevalência dos principais transtornos psiquiátricos, bem como o risco de suicídio e sua gravidade. As entrevistas foram realizadas após as consultas nos ambulatórios supracitados, sem a presença 
de terceiros, em ambiente reservado. Os critérios de exclusão foram: retirar o consentimento e não apresentar capacidade cognitiva para entender os questionários (de acordo com o julgamento clínico do entrevistador e seu supervisor). Os critérios de inclusão foram: ter 18 anos de idade ou mais; assinar o Termo de Consentimento Livre e Esclarecido; ser paciente dos referidos serviços do HUPAA.

\section{Análise dos dados}

A análise estatística foi realizada por meio do pacote estatístico SPSS17. Com base nos dados obtidos, realizou-se análise estatística descritiva, e para correlações, foram utilizados os testes de $X^{2}$, com Intervalo de Confiança de $95 \%$ e valor de $p<0,05$ como padrões de significância. Para a análise da correlação entre transtorno psiquiátrico e gravidade do risco de suicídio, foi utilizado o teste $t$ de Student. Foi realizada uma análise de regressão múltipla para avaliar os quadros de depressão e ansiedade como preditores de risco de suicídio.

\section{RESULTADOS}

\section{Dados sociodemográficos}

Foram entrevistados 253 pacientes, dos quais 42 apresentaram TAG. Desses, 83,3\% eram do sexo feminino, a média de idade foi de $44( \pm 12,2)$ anos e mais da metade era casada $(53,8 \%)$. Ainda entre os pacientes com TAG, a média de escolaridade foi de $7( \pm 4,8)$ anos de estudo (Tabela 1$)$.

\section{Prevalências de TAG nos diferentes ambulatórios investigados}

Cem dos pacientes entrevistados eram do ambulatório de Cardiologia, 85 da Nefrologia e 68 da Psiquiatria. Entre os da Cardiologia, 15\% apresentaram TAG, havendo, aproximadamente, 7\% na Nefrologia e 30,8\% na Psiquiatria. A junção dos ambulatórios não psiquiátricos também revela proporção inferior de TAG $(11,3 \%)$ em relação à Psiquiatria. Considerando toda a amostra, 16,6\% dos pacientes apresentaram TAG (Tabela 1).

\section{Comorbidades psiquiátricas}

A maior parte dos entrevistados com TAG apresentou alguma comorbidade psiquiátrica (88,1\%); $54,8 \%$ possuíam duas ou mais e 33,3\% tinham uma. Os principais transtornos comórbidos com TAG foram os transtornos de humor, particularmente episódios de depressão maior (53,7\%). O risco de suicídio foi observado em 54,8\% dos indivíduos com TAG (Tabela 1).

\section{Risco de suicídio}

Como assinalado anteriormente, o risco de suicídio foi avaliado por meio do MINI 5.0.0. Nas amostras da Cardiologia e da Nefrologia, além da presença desse risco, foi registrada a gravidade desse risco e feita uma análise de correlação com a presença de TAG e/ou depressão/distimia (Tabela 2). De acordo com essa análise, observa-se que a presença de depressão atual aumenta em mais de seis vezes o risco de suicídio, em comparação com quem não apresenta esse transtorno, sendo esse risco de moderado a grave entre os nefropatas/cardiopatas. Já a presença de TAG imprime um risco de suicídio quatro vezes maior que o da população sem TAG, tendendo esse risco a ser de leve gravidade entre os nefropatas/cardiopatas investigados. Quando da comorbidade entre TAG e depressão/distimia, o risco de suicídio é de cerca de seis vezes maior que o da população sem essa combinação de transtornos psiquiátricos, e entre os nefropatas/cardiopatas investigados esse risco é principalmente de moderado a alto.

A análise de regressão múltipla, considerando a presença de TAG e depressão, revelou que, juntos, TAG e depressão foram responsáveis por 29\% da variância na presença de risco de suicídio ( $R$ ajustado de 0,29). O coeficiente de regressão para depressão foi de 0,46 (Intervalo de Confiança de $95 \%=0,34-0,58$ ) e para TAG foi de 0,37 (Intervalo de Confiança de $95 \%=0,19-0,55)$. Os coeficientes de correlação são positivos (para depressão $t=7,5$ e $p<0,01$ e para TAG $t=4,0$ e $p<0,01)$.

Tabela 1. Comparação dos dados sociodemográficos, prevalências ambulatoriais e risco de suicídio entre os pacientes com TAG* e sem TAG deste estudo

\begin{tabular}{|c|c|c|}
\hline Variável avaliada & Sem TAG N (\%) & Com TAG N (\%) \\
\hline Total de pacientes & $211(84,4)$ & $42(16,6)$ \\
\hline \multicolumn{3}{|l|}{ Sexo } \\
\hline Masculino & $81(38,4)$ & $7(16,7)$ \\
\hline Feminino & $130(61,6)$ & $35(83,3)$ \\
\hline Média de idade & $49,5( \pm 14,0)$ & $44( \pm 12,2)$ \\
\hline Escolaridade & $6,2( \pm 4,6)$ & $7,0( \pm 4,8)$ \\
\hline \multicolumn{3}{|l|}{ Estado civil } \\
\hline Casados & $115(55,0)$ & $21(53,8)$ \\
\hline Divorciados & $33(15,8)$ & $8(20,5)$ \\
\hline Solteiros & $34(16,3)$ & $6(15,4)$ \\
\hline Viúvos & $22(10,5)$ & $4(10,3)$ \\
\hline Pacientes do ambulatório de Psiquiatria & $47(69,1)$ & $21(30,8)$ \\
\hline Pacientes dos ambulatórios não psiquiátricos & $164(89,0)$ & $21(11,3)$ \\
\hline Pacientes da Cardiologia & $85(85,0)$ & $15(15,0)$ \\
\hline Pacientes da Nefrologia & $79(92,1)$ & $6(7,0)$ \\
\hline Presença de outro(s) transtorno(s) psiquiátrico(s) & $81(38,4)$ & $37(88,1)$ \\
\hline Um outro transtorno & $52(24,6)$ & $14(33,3)$ \\
\hline Dois ou mais outros transtornos & $29(13,7)$ & $23(54,8)$ \\
\hline Prevalência de depressão maior & $46(21,8)$ & $22(53,7)$ \\
\hline Prevalência de depressão recorrente & $20(9,5)$ & $6(14,6)$ \\
\hline Prevalência de distimia & $17(8,1)$ & $9(21,4)$ \\
\hline Presença de risco de suicídio** & $46(21,8)$ & $23(54,8)$ \\
\hline
\end{tabular}


Tabela 2. Risco de suicídio de acordo com o "status psiquiátrico": presença de transtorno de ansiedade generalizada (TAG) com ou sem depressão/distimia e depressão/distimia sem TAG

\begin{tabular}{|c|c|c|}
\hline Status psiquiátrico & $\begin{array}{l}\text { Avaliação da presença do risco de suicídio } \\
\text { (Teste do qui-quadrado) }\end{array}$ & $\begin{array}{l}\text { Avaliação da gravidade do risco de suicídio } \\
\text { (teste t de Student) }\end{array}$ \\
\hline TAG sem depressão $(\mathrm{N}=19)$ & $\begin{array}{l}\text { - Pacientes com risco: } 11 \\
\begin{array}{c}\chi^{2}=9,6 \\
p<0,01 \\
O R=4,1\end{array}\end{array}$ & $\begin{array}{c}\qquad t=1,8 \\
p=0,07 \\
\text { Tendência de ser risco leve }\end{array}$ \\
\hline TAG com depressão/distimia $(\mathrm{N}=45)$ & $\begin{array}{c}\text { - Pacientes com risco: } 27 \\
\chi^{2}=29,3 \\
p<0,01 \\
O R=5,9\end{array}$ & $\begin{array}{c}t=2,5 \\
\mathrm{p}<0,05 \\
\text { Maioria: risco moderado/grave }\end{array}$ \\
\hline Depressão sem TAG $(\mathrm{N}=47)$ & $\begin{array}{c}\text { - Pacientes com risco: } 29 \\
\chi^{2}=34,2 \\
p<0,01 \\
0 R=6,6\end{array}$ & $\begin{array}{c}t \mathrm{t}=3,3 \\
\mathrm{p}<0,01 \\
\text { Maioria: risco moderado/grave }\end{array}$ \\
\hline Distimia sem TAG $(\mathrm{N}=26)$ & $\begin{array}{c}\text { - Pacientes com risco: } 17 \\
\chi^{2}=21,2 \\
p<0,01 \\
0 R=6,4\end{array}$ & $\begin{array}{c}t=3,4 \\
p<0,01 \\
\text { Mais comum: risco leve }\end{array}$ \\
\hline
\end{tabular}

OR: odds ratio.

\section{DISCUSSÃO}

Houve concordância com estudos anteriores quanto à ocorrência do TAG em relação a sexo, faixa etária e escolaridade ${ }^{17}$, discordando quanto ao estado civil, sendo essa diferença, no entanto, sem significância estatística. A maioria dos estudos relata a presença de TAG principalmente em pessoas sem cônjuge. Grant et al., em seu estudo com 43.093 entrevistados, revelaram que viúvos constituem o grupo mais associado ao TAG ${ }^{18}$.

A amostra com TAG deste estudo teve média de idade de $44( \pm 12,2)$ anos, em semelhança às de estudos anteriores. A média de escolaridade foi também semelhante à dos demais estudos e próxima aos 9,9 anos encontrados no Brasil $^{19}$. O Instituto Brasileiro de Geografia e Estatística (IBGE) também revela que a população brasileira é formada principalmente por pessoas sem cônjuge $(60,1 \%)^{19}$. A população feminina na amostra avaliada teve proporção bastante superior aos 51,3\% encontrados na população geral brasileira, o que reforça a ideia de que o TAG é mais prevalente no sexo feminino ${ }^{19}$.

A prevalência de TAG nas amostras ambulatoriais foi significativamente maior que na população geral. Comparativamente à prevalência na população geral de 2,3\%, encontrada no estudo São Paulo Megacity ${ }^{5}$, o TAG foi quase cinco vezes mais frequente em ambulatórios não psiquiátricos (Cardiologia e Nefrologia), e cerca de 13 vezes mais frequente no ambulatório de Psiquiatria. Torna-se evidente, assim, a associação de TAG com outras condições médicas, psiquiátricas ou não ${ }^{7}$, podendo uma condição agravar a outra e piorar o prognóstico ${ }^{20}$.

A taxa de comorbidade entre TAG e outras condições psiquiátricas é bastante elevada, ocorrendo, na verdade, na maioria dos casos. Um estudo alemão, com 4.181 adultos, revelou comorbidade de $91,3 \%$ de algum transtorno psiquiátrico com o $\mathrm{TAG}^{9}$, resultado semelhante aos $88,1 \%$ do presente estudo; a ocorrência de comorbidade psiquiátrica foi bem menos prevalente em indivíduos sem TAG $(38,4 \%)$. Pesquisa recente, com 12.665 indivíduos, demonstrou inclusive que o TAG é o transtorno mais comórbido com outros transtornos ansiosos, como o transtorno de pânico em 62,3\% dos $\operatorname{casos}^{21}$. A comorbidade pode afetar o funcionamento social e a qualidade de vida das pessoas acometidas. Uma metanálise comprovou que o prejuízo social de indivíduos acometidos por transtornos de humor e TAG foi maior do que nos que apresentavam apenas este transtorno ansioso ${ }^{22}$. Torna-se imprescindível, portanto, investigar comorbidades psiquiátricas ao diagnosticá-lo.

No presente estudo, houve comorbidade predominante com transtornos de humor, principalmente depressão maior. A prevalência desse transtorno foi mais de duas vezes superior em indivíduos com TAG do que naqueles sem esse transtorno. Demonstrou-se previamente que $72 \%$ dos pacientes com TAG ao longo da vida também têm depressão ${ }^{10}$. Outros estudos afirmam que o TAG pode também ser considerado um fator de risco para transtornos depressivos, pois os precede na maioria das ocasiões ${ }^{12}$. Um ano após a ocorrência de TAG, a probabilidade de desenvolver depressão é substancialmente maior do que sem a preexistência desse transtorno ansioso ${ }^{12}$. Sabe-se ainda que quanto mais grave o TAG, maior a chance de surgimento de um quadro depressivo ${ }^{12}$. Alguns pesquisadores reforçam essa correlação ao afirmar que $66,8 \%$ dos indivíduos com TAG apresentam episódio depressivo maior (EDM), enquanto $59,6 \%$ das pessoas com EDM cursam com TAG ${ }^{21}$. Entretanto, enquanto no TAG há preocupação desproporcional e improdutiva com 
eventos futuros, na depressão ocorre afetividade negativa ${ }^{12}$. Além disso, a terapêutica com benzodiazepínicos, utilizada no TAG, não é recomendada em casos de depressão ${ }^{12}$.

O estresse emocional subsequente a uma doença não psiquiátrica pode também contribuir para a ocorrência de TAG em pessoas geneticamente predispostas ${ }^{23}$. Além disso, pessoas com TAG e doença não psiquiátrica podem ter percepção acentuada dos sintomas físicos ${ }^{23}$. Cardiopatas com TAG preexistente, por exemplo, podem ter preocupação excessiva com a condição cardíaca em situações que refletem apenas variações da normalidade, como a ocorrência de taquicardia em momentos estressantes; podem também evitar a prática de exercícios físicos por acreditar ter um coração extremamente frágil, o que, em longo prazo, agravaria a função cardíaca, gerando um círculo vicioso ${ }^{23}$. O TAG pode também gerar alterações diretas na fisiologia cardíaca, na medida em que ocorre uma inibição parassimpática, exacerbando as respostas emocionais aos estímulos do ambiente, o que culmina no aumento da frequência cardíaca ${ }^{23}$. Sendo assim, a variabilidade da frequência cardíaca no repouso torna-se menor em relação a situações de estresse físico ou emocional, as quais efetivamente exigiriam maior débito cardíaco, segundo o modelo de integração neurovisceral já proposto ${ }^{23}$.

Doença renal crônica (DRC) já foi previamente correlacionada com transtornos de ansiedade ${ }^{24}$. No estágio terminal, doentes renais são submetidos à terapia de substituição renal, por transplante renal ou diálise (hemodiálise ou diálise peritoneal); pacientes em hemodiálise, por exemplo, ficam cerca de 4 horas conectados a uma máquina, duas a três vezes por semana, em uma dependência tecnológica e medicamentosa ${ }^{25}$. Essa condição estressante pode propiciar sintomas ansiosos, os quais intensificam a percepção de sintomas físicos comumente relacionados à hemodiálise ${ }^{24}$. A hiperativação simpática, a tensão muscular e a secreção de cortisol subsequente podem aumentar a percepção, por exemplo, da dor e do prurido ${ }^{24}$.

Uma possível consequência negativa dos transtornos de humor é o suicídio, sendo considerado um problema de saúde pública ${ }^{26}$. Segundo estimativas da Organização Mundial de Saúde - OMS (2014), o suicídio figura como a 15 a maior causa de morte no mundo, ocorrendo cerca de 800.000 casos ao ano ${ }^{26}$. Além disso, as tentativas aumentam significativamente o risco de morte por suicídio em tentativas posteriores, constituindo no mais importante fator de risco. Esse risco, se negligenciado pelos profissionais de saúde, pode acarretar o suicídio propriamente dito ${ }^{27}$. A atenção primária exerce papel importante na detecção e manejo precoce do risco de suicídio, já que médicos desse serviço são duas vezes mais procurados que psiquiatras pelos que cometem suicídio ${ }^{27}$. Deve também ser garantida a segurança imediata dos pacientes com maior risco, acompanhada do planejamento terapêutico dos transtornos psiquiátricos as- sociados ${ }^{27}$. Sintomas associados a transtornos de ansiedade são particularmente responsivos à intervenção farmacológica e psicoterapêutica precoce, sendo considerados "fatores de risco modificáveis"14. Thibodeau et al. afirmam que indivíduos com transtornos de ansiedade, como o TAG, possuem maior risco de suicídio, bem como mais tentativas, em relação àqueles sem transtornos de ansiedade ${ }^{13}$.

Este estudo também demonstrou a importância do TAG no comportamento suicida, já que mais da metade dos pacientes com esse transtorno apresentou algum risco de suicídio $(54,8 \%)$, resultado estatisticamente significativo $\left(\chi^{2}=\right.$ 19,2; $p<0,01)$. Uma possível ligação entre TAG e suicidalidade seria a necessidade se anular alguma emoção indesejada, seja tristeza, medo, raiva ou a própria ansiedade ${ }^{13}$. Destaca-se também o prejuízo de "fatores protetores" para o suicídio, como boas interações sociais - acentuando a chance de solidão ${ }^{13}$. Dessa forma, reitera-se que baixo suporte social e prejuízo funcional, provocados pelo transtorno de ansiedade e alta comorbidade com transtorno depressivo, reforçam a ideia de risco aumentado de suicídio entre os pacientes com TAG ${ }^{28}$.

A presença isolada de TAG é suficiente para aumentar o risco de suicídio, mas o risco torna-se aumentado quando há sobreposição com outros transtornos psiquiátricos, como o transtorno depressivo maior - o diagnóstico mais frequente entre os que cometem suicídio ${ }^{29}$. Seja precedendo ou ocorrendo simultaneamente, o fato é que a presença de TAG costuma exacerbar o comportamento suicida em pessoas deprimidas ${ }^{15}$. No presente estudo, no entanto, observou-se que o risco de suicídio é cerca de seis vezes maior com a presença de um quadro depressivo, em comparação àqueles que não apresentam depressão e quatro vezes maior na presença do TAG. Ainda, constatou-se que enquanto a depressão tende a provocar risco de suicídio de moderado a grave, o TAG associa-se a risco leve. Isso pode explicar, pelo menos em parte, por que no presente estudo a associação entre TAG e depressão demonstrou risco de suicídio ligeiramente menor que a ocorrência de depressão isoladamente.

Por outro lado, é importante ressaltar que o presente estudo apresenta limitações, na medida em que a amostra avaliada foi pequena, o que prejudica análises comparativas. Houve também diferença no número de pacientes em cada ambulatório. Outro fator limitador foi a ausência de um grupo-controle saudável para posterior comparação pareada por idade e sexo. Os resultados deste estudo referem-se a investigações realizadas em amostras clínicas de uma instituição pública, não podendo ser generalizáveis para a população geral. Salienta-se, ainda, que os dados foram obtidos por meio de entrevistas realizadas por estudantes de Medicina, que, mesmo treinados, podem estar mais suscetíveis a resultados falsos-positivos que um psiquiatra experiente. O MINI é um instrumento de rastreio, baseado no DSM-IV, que predispõe à sobreposição de sintomas entre os diver- 
sos transtornos psiquiátricos, tais como alterações de sono, concentração e fatigabilidade, presentes tanto em transtornos depressivos quanto em transtornos ansiosos. No DSM-5, inclusive, há um especificador para aqueles pacientes com pelo menos dois sintomas ansiosos durante a maioria dos dias de um episódio depressivo ou de distimia. Ainda assim, segundo Amorim, o MINI pode ser usado para capacitar estudantes quanto a técnicas de avaliação diagnóstica dos transtornos mentais ${ }^{16}$.

\section{CONCLUSÕES}

Os transtornos psiquiátricos são fatores de risco para suicídio. O TAG é um transtorno altamente comórbido com outros transtornos psiquiátricos e com patologias de outras áreas médicas. É notória a correlação entre TAG e transtornos de humor (como a depressão), bem como entre TAG e risco aumentado de suicídio. A presença de TAG em indivíduos com transtorno de humor deve, portanto, ser considerada durante a entrevista psiquiátrica. Torna-se importante, também, investigar o risco de suicídio em indivíduos com TAG/transtornos de humor para prevenir tentativas ou suicídios futuros.

\section{CONTRIBUIÇÕES INDIVIDUAIS}

Juarez Roberto de Oliveira Vasconcelos - Concepção e desenho do estudo, coleta e interpretação dos dados, revisão crítica e aprovação da versão final.

Alice Peixoto da Silva Lôbo - Desenho do estudo, interpretação dos dados, revisão crítica e aprovação da versão final.

Valfrido Leão de Melo Neto - Concepção e desenho do estudo, interpretação dos dados, revisão crítica e aprovação da versão final.

\section{CONFLITOS DE INTERESSE}

Valfrido Leão de Melo Neto recebeu auxílio para educação médica continuada da Daichii Sankyo. Os autores: Juarez Roberto de Oliveira Vasconcelos e Alice Peixoto da Silva Lôbo declaram não possuir conflitos de interesse.

\section{AGRADECIMENTOS}

Agradecemos aos estudantes que auxiliaram na coleta de dados: Dieggo Melo, Ignacio Leutenneger, Susana Almeida, Diego Lisboa, Adolfo Feitosa, Mariana Espíndola, Saulo Cardoso, Stella Farias e Lourena Dantas.

\section{REFERÊNCIAS}

1. Weisberg RB. Overview of generalized anxiety disorder: epidemiology, presentation, and course. J Clin Psychiatry. 2009;70 Suppl 2:4-9.

2. APA Guide to 2013 Annual Meeting. 166th. San Francisco: American Psychiatric Association; 2013.

3. Judd LL, Kessler RC, Paulus MP, Zeller PV, Wittchen HU, Kunovac JL. Comorbidity as a fundamental feature of generalized anxiety disorders: results from the National Comorbidity Study (NCS). Acta Psychiatr Scand Suppl. 1998;393:6-11.

4. Lieb R, Becker E, Altamura C. The epidemiology of generalized anxiety disorder in Europe. Eur Neuropsychopharmacol. 2005;15(4):445-52.

5. Andrade LH, Wang YP, Andreoni S, Silveira CM, Alexandrino-Silva C, Siu ER, et al. Mental disorders in megacities: findings from the São Paulo megacity mental health survey, Brazil. PLoS One. 2012;7(2):e31879.

6. Murcia M, Chastang JF, Niedhammer I. Educational inequalities in major depressive and generalized anxiety disorders: results from the French national SIP study. Soc Psychiatry Psychiatr Epidemiol. 2015;50(6):919-28.

7. Culpepper L. Generalized anxiety disorder and medical illness. J Clin Psychiatry. 2009;70 Suppl 2:20-4.

8. Roy MA, Neale MC, Pedersen NL, Mathé AA, Kendler KS. A twin study of generalized anxiety disorder and major depression. Psychol Med. 1995;25(5):1037-49.

9. Carter RM, Wittchen HU, Pfister H, Kessler RC. One-year prevalence of subthreshold and threshold DSM-IV generalized anxiety disorder in a nationally representative sample. Depress Anxiety. 2001;13(2):78-88.

10. Moffitt TE, Harrington H, Caspi A, Kim-Cohen J, Goldberg D, Gregory AM, et al. Depression and generalized anxiety disorder: cumulative and sequential comorbidity in a birth cohort followed prospectively to age 32 years. Arch Gen Psychiatry. 2007;64(6):651-60.

11. Reckert A, Hinrichs J, Pavenstädt H, Frye B, Heuft G. [Prevalence and correlates of anxiety and depression in patients with end-stage renal disease (ESRD)]. Z Psychosom Med Psychother. 2013;59(2):170-88.

12. Simon NM. Generalized anxiety disorder and psychiatric comorbidities such as depression, bipolar disorder, and substance abuse. J Clin Psychiatry. 2009;70 Suppl 2:10-4.

13. Thibodeau MA, Welch PG, Sareen J, Asmundson GJ. Anxiety disorders are independently associated with suicide ideation and attempts: propensity score matching in two epidemiological samples. Depress Anxiety. 2013;30(10):947-54.

14. Fawcett J, Scheftner WA, Fogg L, Clark DC, Young MA, Hedeker D, et al. Time-related predictors of suicide in major affective disorder. Am J Psychiatry. 1990;147(9):1189-94.

15. Thaipisuttikul P, Ittasakul P, Waleeprakhon P, Wisajun P, Jullagate S. Psychiatric comorbidities in patients with major depressive disorder. Neuropsychiatr Dis Treat. 2014;10:2097-103.

16. Amorim P. Mini International Neuropsychiatric Interview (MINI): validação de entrevista breve para o diagnóstico de transtornos mentais. Rev Bras Psiquiatr. 2000;22(3):106-15.

17. Chazelle E, Lemogne C, Morgan K, Kelleher CC, Chastang JF, Niedhammer I. Explanations of educational differences in major depression and generalised anxiety disorder in the Irish population. J Affect Disord. 2011;134(1-3):304-14.

18. Grant BF, Goldstein RB, Chou SP, Huang B, Stinson FS, Dawson DA, et al. Sociodemographic and psychopathologic predictors of first incidence of DSM-IV substance use, mood and anxiety disorders: results from the Wave 2 National Epidemiologic Survey on Alcohol and Related Conditions. Mol Psychiatry. 2009;14(11):1051-66.

19. Censo demográfico 2010. IBGE [online]. Rio de Janeiro, Brasil; 2011. Disponível em: <http:// www.ibge.gov.br/home/estatistica/populacao/censo2010>. Acesso em: 13 set. 2015.

20. Combs H, Markman J. Anxiety disorders in primary care. Med Clin North Am. 2014;98:1007-23.

21. Al-Asadi AM, Klein B, Meyer D. Multiple comorbidities of 21 psychological disorders and relationships with psychosocial variables: a study of the online assessment and diagnostic system within a web-based population. J Med Internet Res. 2015;17(2):e55.

22. Hoffman DL, Dukes EM, Wittchen HU. Human and economic burden of generalized anxiety disorder. Depress Anxiety. 2008;25(1):72-90.

23. Thayer JF, Lane RD. A model of neurovisceral integration in emotion regulation and dysregulation. J Affect Disord. 2000;61(3):201-16. 
24. Perales-Montilla CM, Duschek S, Reyes-Del Paso GA. The influence of emotional factors on the report of somatic symptoms in patients on chronic haemodialysis: the importance of anxiety. Nefrologia. 2013;33(6):816-25.

25. Páez AE, Jofré MJ, Azpiroz (R, Bartoli MA. Ansiedad y depresión en pacientes con insuficiencia renal crónica en tratamiento de diálisis. Univ Psychol. 2008;8(1):117-24.

26. WHO. Preventing suicide: a global imperative. Geneva: World Health Organization (WHO); 2014.
27. Luoma JB, Martin CE, Pearson JL. Contact with mental health and primary care providers before suicide: a review of the evidence. Am J Psychiatry. 2002;159(6):909-16.

28. Bomyea J, Lang AJ, Craske MG, Chavira D, Sherbourne CD, Rose RD, et al. Suicidal ideation and risk factors in primary care patients with anxiety disorders. Psychiatry Res. 2013:209(1):60-5.

29. Kessler RC, Borges $G$, Walters EE. Prevalence of and risk factors for lifetime suicide attempts in the National Comorbidity Survey. Arch Gen Psychiatry. 1999;56(7):617-26 